\title{
Electromagnetic Induction at a Model Ocean Coast ${ }^{\dagger}$
}

\author{
G. Fischer, * P.-A. SCHNEGG, ${ }^{*}$ and K. D. UsAdeL** \\ * Observatoire Cantonal, Neuchâtel, Switzerland \\ **Gesamthochschule, Fachbereich, Duisburg, GFR
}

(Received November 28, 1977; Revised February 2, 1978)

The electromagnetic response of an ocean coast model to a vertically incident monochromatic plane wave is studied. The model consists of a perfectly conducting half-plane (the model ocean) resting at the surface of a good conductor (the model earth). Values of electric and magnetic field components at the surfaces of land and ocean, as well as at the ocean floor, are given in tabular form for both $E$ - and $H$ polarization induction.

We have calculated the $E$-polarization electromagnetic response to a vertically incident monochromatic plane wave of a two-dimensional ocean coast model consisting of a perfectly conducting half-plane (the model ocean) resting at the surface of a conductor (the model earth) with which it is in good contact. This model, as well as the coordinate system chosen, is shown in Fig. 1. Our method of solution is analytical and strictly rigorous up to the point where the electric surface field is expressed in terms of an integral equation. This equation is then solved numerically. A detailed description of this work has been published elsewhere (FISCHER et al., 1978).

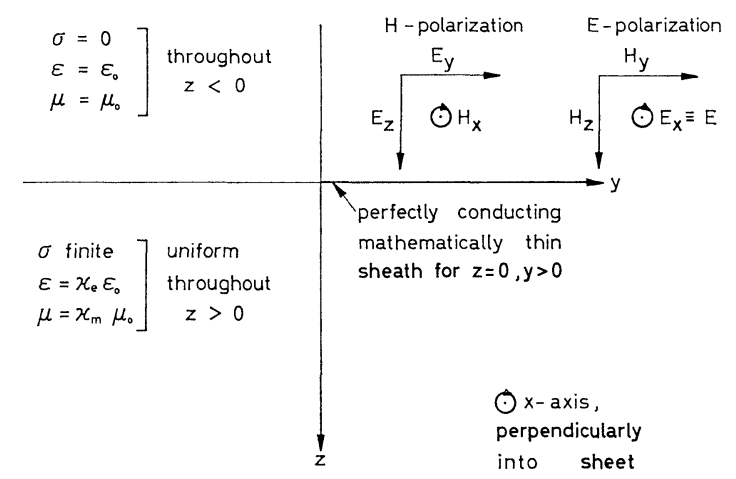

Fig. 1. Cross-section of the conductive two-dimensional structure chosen as model of an ocean coast. The mathematically thin perfect conductor at $(z=0, y>0)$ represents the ocean. A monochromatic plane wave is incident vertically on this structure. For $H$-polarization induction the only field components concerned are $E_{y}, E_{z}$, and $H_{x}$. With $E$-polarization the components involved are $E_{x}, H_{y}$, and $H_{z}$.

t The authors wish to dedicate this article to Professor Jean Rossel on the occasion of his 60th birthday. 
Since there is at present a great deal of interest in comparing the predictions of different numerical methods, as well as those of analog laboratory scale models, with the results of analytical calculations, we present our results in the form of a table of numerical data. To facilitate this kind of comparison we also present a table of similar data for $H$-polarization induction, derived from an entirely analytical solutions recently published by BAILeY (1977) and NiCOLL and WeAver (1977). These Tables 1 and 2 refer to field component values at the $z=0$ surface of the model of Fig. 1 , in the quasistatic $(\omega \varepsilon / \sigma \ll 1)$ and non-magnetic $\left(\mu \equiv \mu_{0}\right)$ limit. Distances along the surface $(y$-coordinate) are expressed in skin-depths $\delta$,

Table 1. Values of electric and magnetic fields at the surfaces of land and ocean $(z=-0)$, as well as at the ocean floor $(y>0, z=+0)$, for $E$-polarization induction in the model structure of Fig. 1.

\begin{tabular}{|c|c|c|c|c|c|c|c|c|}
\hline$y$ & $\begin{array}{c}\operatorname{Re} E_{x} \\
z= \pm 0\end{array}$ & $\begin{array}{c}\operatorname{Im} E_{x} \\
z= \pm 0\end{array}$ & $\begin{array}{c}\mathrm{Re} H_{y} \\
z=-0\end{array}$ & $\begin{array}{c}\operatorname{Im} H_{y} \\
z=-0\end{array}$ & $\begin{array}{c}\mathrm{Re} H_{y} \\
z=+0\end{array}$ & $\begin{array}{c}\operatorname{Im} H_{y} \\
z=+0\end{array}$ & $\begin{array}{c}\operatorname{Re} H_{z} \\
z= \pm 0\end{array}$ & $\begin{array}{c}\operatorname{Im} H_{z} \\
z= \pm 0\end{array}$ \\
\hline$-\infty$ & 1 & 1 & 1 & 0 & 1 & 0 & 0 & 0 \\
\hline-8 & .9899 & 1.0036 & .981 & .023 & .981 & .023 & .001 & .003 \\
\hline-7 & .9828 & 1.0053 & .972 & .032 & .972 & .032 & .001 & .004 \\
\hline-6 & .9735 & 1.0071 & .959 & .044 & .959 & .004 & .001 & .006 \\
\hline-5 & .9601 & 1.0100 & .941 & .062 & .941 & .062 & .002 & .008 \\
\hline-4 & .9382 & 1.0151 & .911 & .090 & .911 & .900 & .003 & .015 \\
\hline-3 & .8961 & 1.0229 & .851 & .136 & .851 & .136 & .004 & .030 \\
\hline-2 & .8031 & 1.0217 & .709 & .201 & .709 & .201 & -.009 & .068 \\
\hline-1 & .5925 & .9339 & .360 & .218 & .360 & .218 & -.104 & .153 \\
\hline-.8 & .5264 & .8836 & .244 & .196 & .244 & .196 & -.151 & .179 \\
\hline-.6 & .4489 & .8102 & .102 & .150 & .102 & .150 & -.222 & .210 \\
\hline-.4 & .3568 & .7002 & -.071 & .070 & -.071 & .070 & -.340 & .253 \\
\hline-.2 & .2414 & .5224 & -.302 & -.075 & -.302 & -.075 & -.592 & .337 \\
\hline-.1 & .1643 & .3759 & -.436 & -.204 & -.436 & -.204 & -.942 & .459 \\
\hline-0 & 0 & 0 & -0.57 & 0.00 & -0.57 & 0.00 & $-\infty$ & $+\infty$ \\
\hline+0 & 0 & 0 & $+\infty$ & $-\infty$ & $-\infty$ & $+\infty$ & 0 & 0 \\
\hline+.1 & 0 & 0 & 1.520 & -.305 & -.253 & .273 & 0 & 0 \\
\hline .2 & 0 & 0 & 1.374 & -.231 & -.128 & .188 & 0 & 0 \\
\hline .4 & 0 & 0 & 1.245 & -.163 & -.037 & .105 & 0 & 0 \\
\hline .6 & 0 & 0 & 1.184 & -.128 & -.0057 & .063 & 0 & 0 \\
\hline .8 & 0 & 0 & 1.148 & -.107 & +.0057 & .039 & 0 & 0 \\
\hline 1 & 0 & 0 & 1.124 & -.092 & .0093 & .024 & 0 & 0 \\
\hline 2 & 0 & 0 & 1.069 & -.056 & .0043 & .0005 & 0 & 0 \\
\hline 3 & 0 & 0 & 1.048 & -.041 & .0006 & -.0008 & 0 & 0 \\
\hline 4 & 0 & 0 & 1.037 & -.032 & -.0001 & -.0002 & 0 & 0 \\
\hline 5 & 0 & 0 & 1.030 & -.027 & -.00005 & -0 & 0 & 0 \\
\hline 6 & 0 & 0 & 1.025 & -.023 & -0 & +0 & 0 & 0 \\
\hline 7 & 0 & 0 & 1.022 & -.020 & +0 & +0 & 0 & 0 \\
\hline 8 & 0 & 0 & 1.019 & -.018 & +0 & +0 & 0 & 0 \\
\hline$+\infty$ & 0 & 0 & 1 & 0 & 0 & 0 & 0 & 0 \\
\hline
\end{tabular}

The electric field parameters are normalized to the value of $\operatorname{Re} E_{x}$ at $y=-\infty$. All magnetic field components are normalized to $\operatorname{Re} H_{y}$ at $y=-\infty$. Coordinate $y$ is expressed in skin depths. These data are for the quasi-static and non-magnetic limit, and were derived from FISCHER et al. (1978). 


$$
\delta=\left(\frac{2}{\omega \mu_{0} \sigma}\right)^{1 / 2}
$$

Real (in phase) and imaginary (in quadrature) parts of electric fields are normalized to the

Table 2. Values of electric and magnetic fields at the surfaces of land and ocean $(z=-0)$, at well as at the ocean floor $(y>0, z=+0)$ for $H$-polarization induction in the model structure of Fig. 1.

\begin{tabular}{|c|c|c|c|c|c|c|}
\hline$y$ & $\begin{array}{c}\operatorname{Re} E_{y} \\
z= \pm 0\end{array}$ & $\begin{array}{c}\operatorname{Im} E_{y} \\
z= \pm 0\end{array}$ & $\begin{array}{c}\operatorname{Re} E_{z} \\
z=+0\end{array}$ & $\begin{array}{c}\operatorname{Im} E_{z} \\
z=+0\end{array}$ & $\begin{array}{c}\operatorname{Re} H_{x} \\
z=+0\end{array}$ & $\begin{array}{c}\operatorname{Im} H_{x} \\
z=+0\end{array}$ \\
\hline$-\infty$ & 1 & 1 & 0 & 0 & 1 & 0 \\
\hline-4 & .9998 & 1.0004 & 0 & 0 & 1 & 0 \\
\hline-3 & .9982 & 1.0002 & 0 & 0 & 1 & 0 \\
\hline-2 & .9952 & .9932 & 0 & 0 & 1 & 0 \\
\hline-1.5 & .9985 & .9807 & 0 & 0 & 1 & 0 \\
\hline-1 & 1.0234 & .9544 & 0 & 0 & 1 & 0 \\
\hline-.8 & 1.0518 & .9389 & 0 & 0 & 1 & 0 \\
\hline-.6 & 1.1073 & .9220 & 0 & 0 & 1 & 0 \\
\hline-.5 & 1.1545 & .9146 & 0 & 0 & 1 & 0 \\
\hline-.4 & 1.2261 & .9108 & 0 & 0 & 1 & 0 \\
\hline-.3 & 1.3420 & .9162 & 0 & 0 & 1 & 0 \\
\hline-.2 & 1.5551 & .9481 & 0 & 0 & 1 & 0 \\
\hline-.1 & 2.0775 & 1.0827 & 0 & 0 & 1 & 0 \\
\hline-.08 & 2.2961 & 1.1511 & 0 & 0 & 1 & 0 \\
\hline-.06 & 2.6207 & 1.2599 & 0 & 0 & 1 & 0 \\
\hline-.04 & 3.1726 & 1.4575 & 0 & 0 & 1 & 0 \\
\hline-.02 & 4.4347 & 1.9389 & 0 & 0 & 1 & 0 \\
\hline-0 & $+\infty$ & $+\infty$ & 0 & 0 & 1 & 0 \\
\hline+0 & 0 & 0 & $+\infty$ & $+\infty$ & 1 & 0 \\
\hline .02 & 0 & 0 & 4.3311 & 1.6933 & .8254 & -.0710 \\
\hline .04 & 0 & 0 & 3.0247 & 1.1134 & .7540 & -.0981 \\
\hline .06 & 0 & 0 & 2.4381 & .8425 & .7000 & -.1174 \\
\hline .08 & 0 & 0 & 2.0836 & .6736 & .6550 & -.1325 \\
\hline .1 & 0 & 0 & 1.8381 & .5539 & .6159 & -.1447 \\
\hline .2 & 0 & 0 & 1.2056 & .2352 & .4688 & -.1817 \\
\hline .3 & 0 & 0 & .9036 & .0840 & .3647 & -.1969 \\
\hline .4 & 0 & 0 & .7111 & -.0052 & .2845 & -.2005 \\
\hline .5 & 0 & 0 & .5722 & -.0616 & .2207 & -.1970 \\
\hline .6 & 0 & 0 & .4652 & -.0978 & .1690 & -.1888 \\
\hline .8 & 0 & 0 & .3095 & -.1335 & .0926 & -.1651 \\
\hline 1 & 0 & 0 & .2027 & -.1409 & .0420 & -.1373 \\
\hline 1.5 & 0 & 0 & .0546 & -.1093 & -.0169 & -.0731 \\
\hline 2 & 0 & 0 & -.0023 & -.0642 & -.0275 & -.0299 \\
\hline 3 & 0 & 0 & -.0166 & -.0098 & -.0124 & +.0024 \\
\hline 4 & 0 & 0 & -.0055 & +.0028 & -.0015 & .0038 \\
\hline$+\infty$ & 0 & 0 & 0 & 0 & 0 & 0 \\
\hline
\end{tabular}

All electric field components are normalized to the value of $\operatorname{Re} E_{y}$ at $y=-\infty$. The magnetic field parameters at the ocean floor $H_{x}(y>0, z=+0)$ are normalized to the constant surface field $H_{x}(z=-0)$. Note that for $z=-0$ one has $E_{z}=0$ when $y>0$. Coordinate $y$ is expressed in skin-depths. These data are for the quasi-static and non-magnetic limit, and were derived from BAILEY (1977) and NiCOLL and WEAVER (1977). 
values of $\operatorname{Re} E_{x}$ (E-polarization) or $\operatorname{Re} E_{y}$ ( $H$-polarization) at $z=0, y=-\infty$. Magnetic fields are normalized in similar fashion to $\operatorname{Re} H_{y}$ and $\operatorname{Re} H_{x}$, respectively. The fields $H_{y}$ and $H_{x}$ at $(z=0, y=-\infty)$ are taken as phase references and therefore chosen to be purely real.

The true complex ratios of $E_{x}$ to $H_{y}(E$-polarization, Table 1$)$ and of $-E_{y}$ to $H_{x}(H$ polarization, Table 2) are in fact given by the surface impedances $Z_{x y}$ and $-Z_{y x}$. At $(z=0, y=-\infty)$ these ratios are identical,

$$
Z_{x y}(-\infty)=-Z_{y x}(-\infty)=(1+\mathrm{i})\left(\frac{\omega \mu_{0}}{2 \sigma}\right)^{1 / 2} .
$$

In the present geometry and coordinate system it is easy to show (see, e.g., Fischer, 1975) that

$$
Z_{x x}=Z_{y y}=0
$$

throughout the structure. Note also that for $H$-polarization, with the normalization convention and in the limit stated, $H_{x}=1$ at the surfaces of both land and ocean $(z=-0)$, and is therefore not tabulated for $z=-0$. Also not tabulated is $E_{z}(y<0)$ at $z=-0$, since this field will be generated by surface charges that build up at the surface. The currents required to build and carry away these surface charges are small, i.e. of order $\omega \varepsilon / \sigma$ (c.f. PRICE, 1973), but the highly singular nature of our model ocean edge may result in significant vertical fields near the coast, to satisfy div $\mathrm{E}=0$. At $(y>0, z=-0), E_{z} \equiv 0$ however.

In Fig. 2 we give a representation of the surface impedance in terms of their real

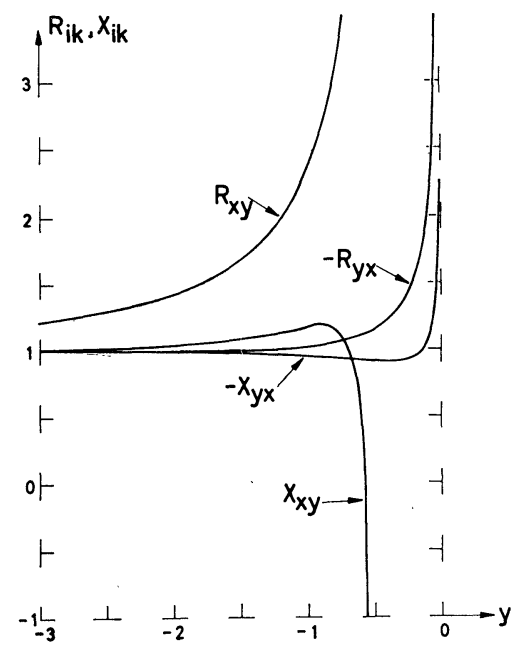

Fig. 2. Real and imaginary parts of the surface impedances for the structural model of Fig. 1. $Z_{x y}=R_{x y}+\mathrm{i} X_{x y}$ refers to $E$-polarization and $Z_{y x}=R_{y x}+\mathrm{i} X_{y x}$ to $H$-polarization induction. Units of $y$ coordinate are skin-depths, while those of $R_{i k}$ and $X_{i k}$ are defined by Eq. (2). Close to the origin $R_{x y}$ and $X_{x y}$ rather suddenly, though continuously, vanish (this is not represented), whereas both $R_{y x}$ and $X_{y x}$ tend toward $-\infty$. For $y>0, Z_{x y}$ and $Z_{y x}$ both vanish, and in this geometry and coordinate system $Z_{x x}$ and $Z_{y y}$ are nil throughout the structure. 
and imaginary parts,

$$
Z_{i k}=R_{i k}+\mathrm{i} X_{i k},
$$

where the $(i k)$ subscript pair stands for $(x y)$ or $(y x)$. The most striking feature of Fig. 2 is the much larger distance to which the ocean coast effect is carried into the land for $E$-polarization than for $H$-polarization induction. As shown in more detail elsewhere (FISCHER et al., 1978), this arises because the integrated ocean current $I_{x}$ (current per unit length of ocean sheet, given by $\left.I_{x}=H_{y}(z=-0)-H_{y}(z=+0)\right)$ increases to very large values as the seashore is approached. In our idealized ocean model (Fig. 1) this integrated current even goes to infinity at the ocean edge $(y=+0, z=0)$, where it acts as a very long lineantenna radiating the coast effect far afield. Under $H$-polarization induction the total integrated current (from $z=0$ to $z=+\infty$ ) is a constant, for reasons of continuity, with the consequent constancy of the surface magnetic field $H_{x}$ as said above. Here the integrated ocean current $I_{y}=H_{x}(z=+0)-H_{x}(z=-0)$ increases monotonically from zero at the seashore to this total constant value as $y \rightarrow+\infty$. There is no line-antenna feature, and the coast effect is not carried to very large distances under $H$-polarization induction. Similar differences for the ranges to which perturbations are carried in the two polarizations can be expected for other two-dimensional structures, whenever large conductivity contrasts are encountered at shallow depths.

This work was supported in part by a grant from the Swiss National Science Foundation.

\section{REFERENCES}

BAILEY, R. C., Electromagnetic induction over the edge of a perfectly conducting ocean: the $H$-polarization case, Geophys. J. R. Astr. Soc., 48, 385-392, 1977.

FISCHER, G., Symmetry properties of the surface impedance tensor for structures with a vertical plane of symmetry, Geophysics, 40, 1046-1050, 1975.

Fischer, G., P.-A. SchNegG, and K. D. UsADel, Electromagnetic response of an ocean coast model to E-polarization induction, Geophys. J. R. Astr. Soc., 52, 599-616, 1978.

Nicoll, M. A. and J. T. WEAVER, $H$-polarization induction over an ocean edge coupled to the mantle by a conducting crust, Geophys. J. R. Astr. Soc., 49, 427-441, 1977.

Price, A. T., The theory of geomagnetic induction, Phys. Earth Planet. Inter., 7, 227-233, 1973. 